Document downloaded from:

http://hdl.handle.net/10251/179796

This paper must be cited as:

Molins-Benlliure, J.; Cabedo Fabres, M.; Antonino Daviu, E.; Ferrando Bataller, M. (2020). Effect of the Size and Shape of the Ground Plane in Small Antennas Efficiency. IEEE. 1-4. https://doi.org/10.1109/iWAT48004.2020.1570615436

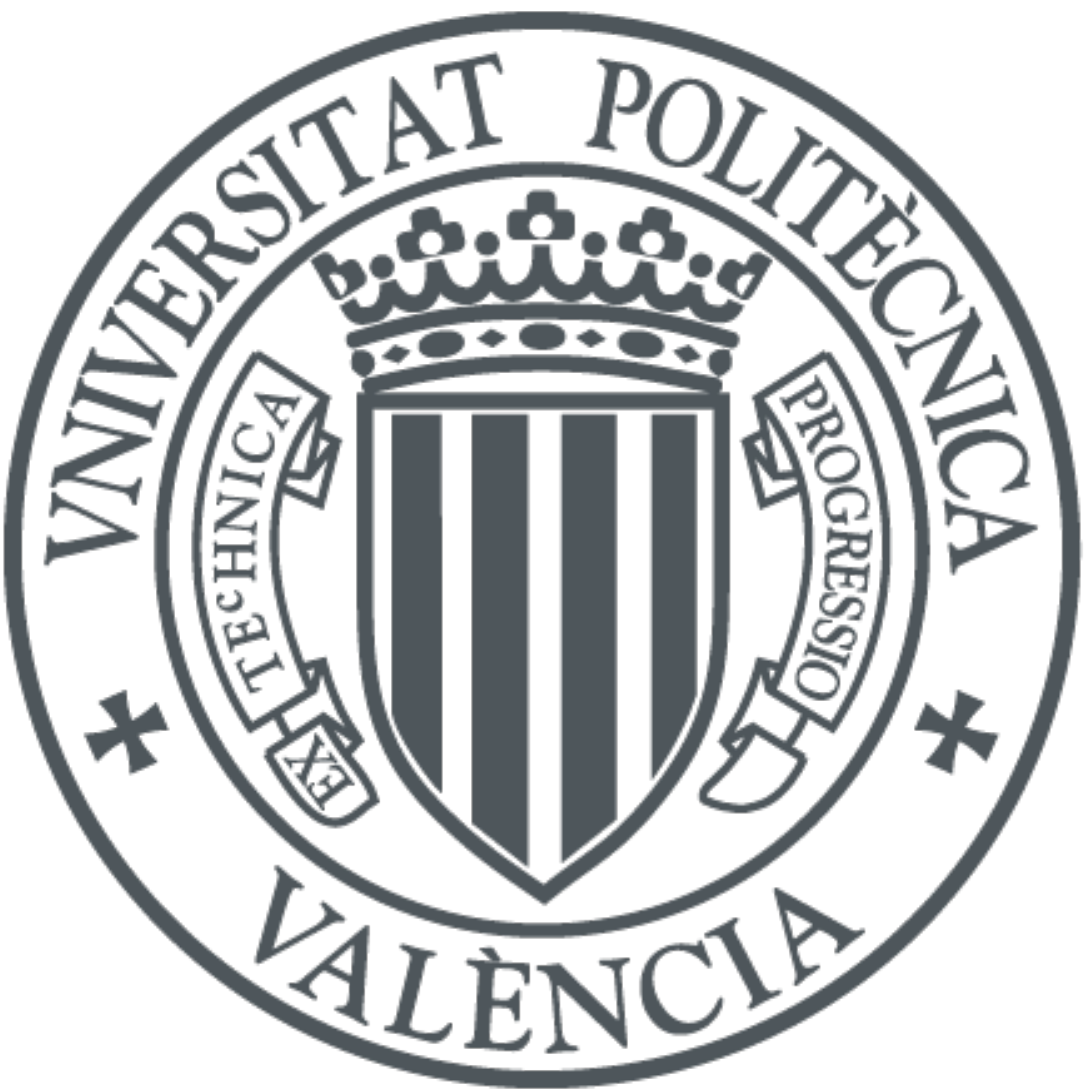

The final publication is available at

https://doi.org/10.1109/iWAT48004.2020.1570615436

Copyright IEEE

Additional Information 


\title{
Effect of the Size and Shape of the Ground Plane in Small Antennas Efficiency
}

\author{
Jaime Molins-Benlliure, Marta Cabedo-Fabrés, Eva Antonino-Daviu, Miguel Ferrando-Bataller \\ Instituto de Telecomunicaciones y Aplicacionens Multimedia (iTEAM) \\ Universitat Politècnica de València \\ València, Spain \\ jaimoben@iteam.upv.es
}

\begin{abstract}
An analysis of the radiation efficiency depending on the position of a small antenna and the size and shape of the ground plane is presented. For the analysis a LTCC based chip antenna has been used placing it on FR4 square, circular and triangular PCBs. The study is an insight of the key points for obtaining proper radiation efficiency in designs for the new licensed 5G IoT bands (600 MHz-USA, 700 MHz-Europe) and the ISM bands (868 MHz-Europe, 915 MHz-USA).

Index Terms-small antenna, IoT, chip antenna, ground plane effect, radiation efficiency
\end{abstract}

\section{INTRODUCTION}

The scenario of all connected devices, fueled by the internet of things (IoT) concept, has triggered the demand of small antennas specially for the sub-1 $\mathrm{GHz}$ bands including the new licensed 5G [1] bands for wide area deployments (600 MHz-USA, $700 \mathrm{MHz}$-Europe) and the ISM bands (868 MHz-Europe, $915 \mathrm{MHz}-U S A$ ).

The size of some handheld devices brings the challenge of including an antenna in such a limited space. Antenna miniaturization is limited by the length of $\lambda / 4$ corresponding to a resonant monopole. For the lowest frequency bands of IoT, $600 \mathrm{MHz}$, the wavelength is $\lambda=500 \mathrm{~mm}$, and a resonating monopole at that frequency has a length of $\lambda / 4=125 \mathrm{~mm}$.

Including an antenna of $125 \mathrm{~mm}$ length or similar in a small device [2] [3] is a really challenging task, specially, when the device manufacturer leave a limited area for the antenna. The most common solutions are the inverted L (ILA) and the inverted F (IFA) [4] antennas which are based on a tilted monopole, but in some cases, further miniaturization techniques must be added.

Some miniaturization solutions are used such as meandering or antenna loading (add a capacitance or inductance to the antenna). Besides, there exists chip antennas from companies like Johanson [5], Antenova [6] or Fractus [7] which provides an embedded antenna in a ceramic substrate, being able to get a low frequency antenna with an extremely limited size ceramic chip.

This work has been supported by the Spanish Ministry of Science, Innovation, and Universities (Ministerio de Ciencia, Innovación y Universidades) under the research program (TEC2016-78028-C3-3-P).
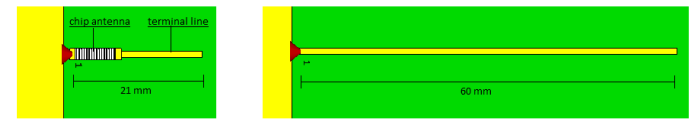

(a)
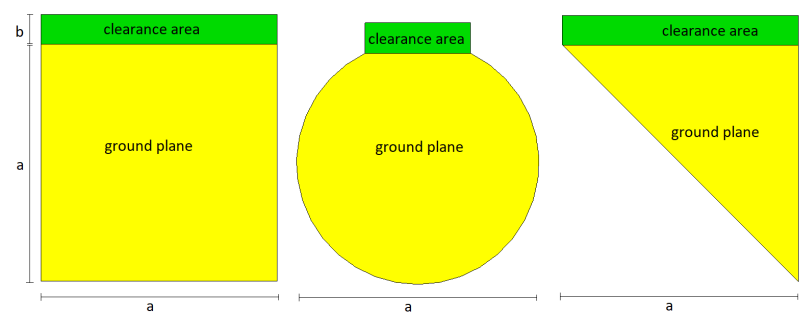

(b)

Fig. 1. a) Comparison between chip antenna+terminal line and monopole antenna and, b) Square, round and triangular PCB

Small antennas are limited in terms of radiation properties [8]- [11], and the main contribution of the radiation is produced by the ground plane. The size of the ground plane has an enormous impact in the antenna bandwidth and efficiency, therefore it is really important to respect a minimum size in order not to limit the performance of the antenna. Because of this feature, this kind of solutions must take into account both parts of the structure, the monopole and the ground plane, thus, it must be considered as an asymmetric dipole where one arm is the antenna and the other arm is the ground plane. Moreover, the position of the antenna and its surroundings have also an important role, and in this paper, an analysis of the positioning of the antenna depending on the size and shape of the ground plane has been realized.

The radiation efficiency of the antenna in three different FR4 $\left(\epsilon_{r}=4.6, \tan \delta=0.0035, t=1.6 \mathrm{~mm}\right) \mathrm{PCB}$ has been studied, with squared, round and triangular shape (Fig. 1) modifying their size and the position of the antenna. 


\section{Antenna Design}

For the ground plane analysis a chip antenna connected in series with a terminal line has been used. The chip antenna resonates at $1.4 \mathrm{GHz}$, but connecting in series the terminal line, the antenna can be tuned at lower frequencies modifying the length of the line. In this paper, the design has been tuned at $868 \mathrm{MHz}$ (European ISM Band), but it could be tuned for any sub-1GHz $5 \mathrm{G}$ band or $915 \mathrm{MHz}$ ISM band (USA).

The chip antenna has been designed with a coil inside a ceramic substrate $\left(\epsilon_{r}=7.1\right.$ and $\left.\tan \delta=0.0009\right)$ to be fabricated in LTCC technology with a dimension of $8.22 \times 1.8 \times 0.6 \mathrm{~mm}$ and the connected line length is 13 $\mathrm{mm}$. Loading the design with a coil allows to shorten an antenna length, maintaining the resonance frequency, due to the series inductance which compensates the capacitive behaviour when shorten a monopole. In the Fig. 1 a comparison between a resonating monopole, and the proposed design is depicted.

\section{GRound Plane Analysis}

In this section, a study of the radiation efficiency of the proposed antenna depending on its position and the size and shape of the ground plane is presented. For the analysis, three different FR4 based PCB has been used, with square, circular and triangular shape (Fig. 1 (b)).

\section{A. Square Ground Plane}

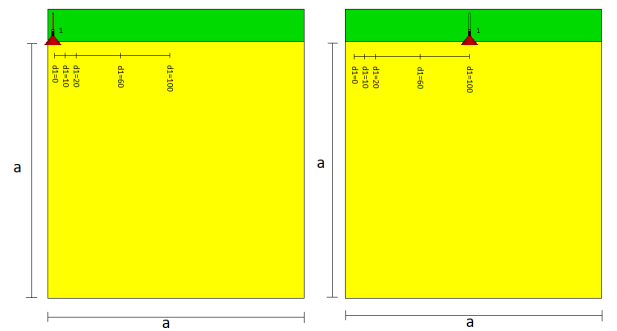

(a)

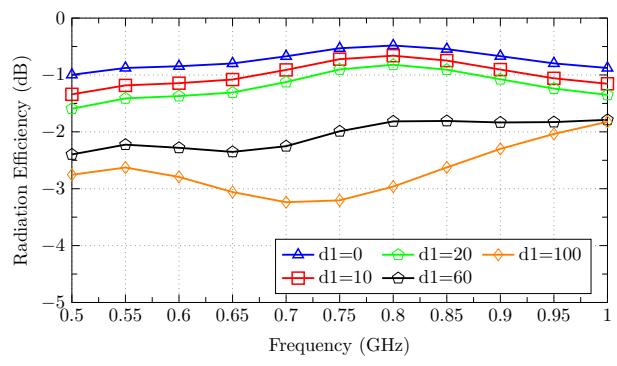

(b)

Fig. 2. a) Left: Design with the antenna placed in the corner $(\mathrm{d} 1=0)$, Right: Design with the antenna placed in the center $(\mathrm{d} 1=100)$ and, b) Radiation Efficiency obtained displacing the antenna from $\mathrm{d} 1=0$ until $\mathrm{d} 1=100$

The first study is based on the squared PCB. In order to check the optimum position for the antenna in terms of radiation efficiency, an analysis in a $\mathrm{PCB}$ with a size of $\mathrm{a}=200 \mathrm{~mm}$ has been done. The antenna has been displaced through the $\mathrm{x}$ axis from the corner $(\mathrm{d} 1=0$ Fig. 2$)$ until the center $(\mathrm{d} 1=100 \mathrm{~mm}$ Fig. 2$)$ of the upper side of the PCB. In Fig. 2 b), results show a decrease of radiation efficiency when the antenna approaches the center position $(\mathrm{d} 1=100$ $\mathrm{mm}$ ), and on the other hand, the best results are obtained placing the antenna in the corner( $\mathrm{d} 1=0 \mathrm{~mm})$.

Once the optimum position of the antenna has been fixed (d1=0 Fig. 2), an analysis of the size of the ground plane is realized. In Fig. 2 (b), the radiation efficiency for a PCB from a size of $\mathrm{a}=30 \mathrm{~mm}$ up to $\mathrm{a}=200 \mathrm{~mm}$ is depicted. Results shows an increment of radiation efficiency with the size of the PCB. If results are analyzed in detail, when the length of the ground plane (a) is higher than $\lambda / 2(172 \mathrm{~mm})$, the increase of radiation efficiency is saturated, as seen in the case of $\mathrm{a}=172,200 \mathrm{~mm}$.

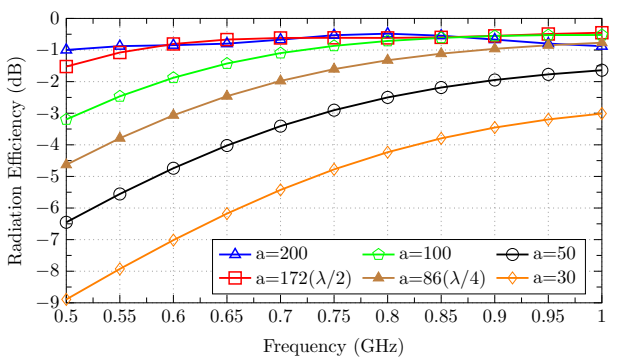

(a)

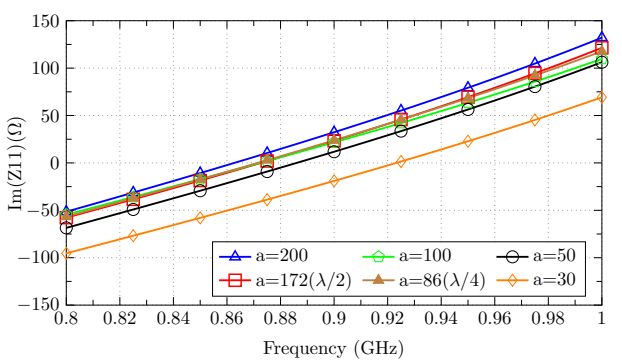

(b)

Fig. 3. a) Radiation efficiency of the antenna in the square PCB modifying the side length of the ground plane (a) and, b) $\operatorname{Re}(Z 11)$ of the antenna in the square PCB modifying the side length of the ground plane (a)

Further analysis is shown in Fig. 3 (b) with the $\operatorname{Im}(\mathrm{Z} 11)$. The resonance frequency is stable when the side length (a) of the PCB is close or higher than $\lambda / 4(86 \mathrm{~mm})$. In case the side length (a) is shorter than $\lambda / 4$, the resonance frequency is shifted toward higher frequencies. As said in the introduction, small antennas are totally dependent to their connected ground plane, and they must be considered as a asymmetric dipole. In a asymmetric dipole, the shorter arm, limits the resonance, therefore, when the ground plane is shorter than $\lambda / 4$, the resonance is displaced to higher frequencies. 


\section{B. Circular Ground Plane}

The second study is based on a circular PCB for comparing the results with the square one. In Fig. 4 (a) the PCB is shown. It has a circular ground plane, but on top, a rectangular clearance area of FR4 has been settled for placing the antenna.

As the previous study, an analysis of the position of the antenna has been made. A PCB of $\mathrm{a}=200 \mathrm{~mm}$ has been fixed. In Fig. 4 (b) the radiation efficiency is depicted, displacing the antenna from the corner of the clearance area until the center. The radiation efficiency is higher when the antenna is closer to the corner, and corroborate the results of the square PCB analysis.
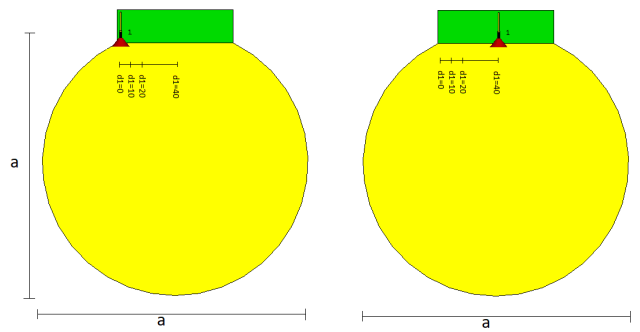

(a)

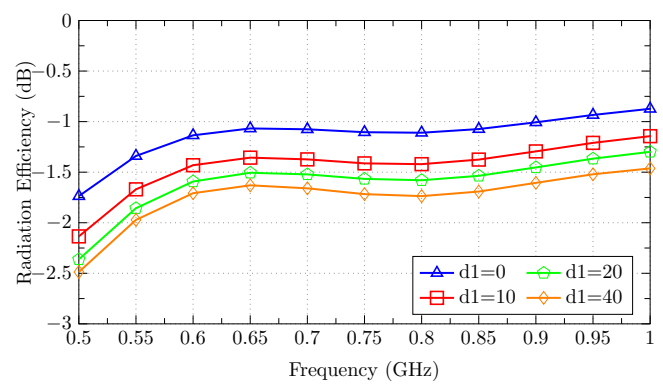

(b)

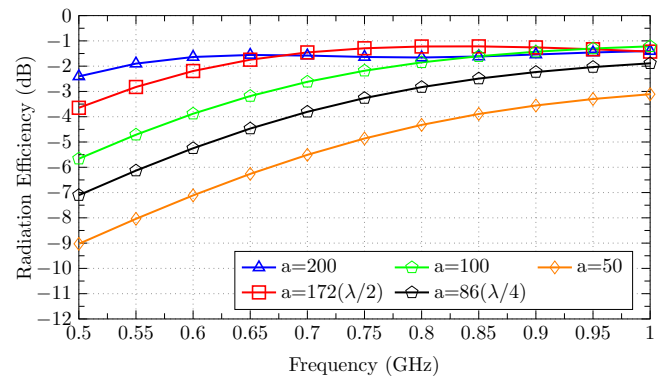

(c)

Fig. 4. a) Left: Round PCB with the antenna placed in the corner $(\mathrm{d} 1=0)$, Right: Round PCB with the antenna placed in the center(d1=100), b)Radiation Efficiency obtained displacing the antenna from $\mathrm{d} 1=0$ until $\mathrm{d} 1=100$ and, c) Radiation efficiency of the antenna in the round PCB modifying the side length of the ground plane (a)

In Fig. 4 (c) the study of increasing the size of the ground plane is depicted. As seen in the previous PCB, the efficiency increases with the size of the ground plane(a) and it saturates at when $\mathrm{a}=\lambda / 2$.

\section{Triangular Ground Plane}

The last study is based on the triangular shape PCB. In this case, the antenna has been placed in one of the cathetus, moving it from the acute angle corner $(\mathrm{d} 1=0)$ until the right angle $(\mathrm{d} 1=200)$. In this case an analysis from angle to angle must be done due to the non-symmetric structure. Fig. 5 (b) shows the radiation efficiency results, and it can be observed that the worst scenario is when the antenna is in the middle $(\mathrm{d} 1=100)$, then when the antenna is on the corners, the radiation efficiency is higher, but in the case of the acute angle corner $(\mathrm{d} 1=0)$ the efficiency remains more stable with frequency than in the case of the right angle $(\mathrm{d} 1=200)$.

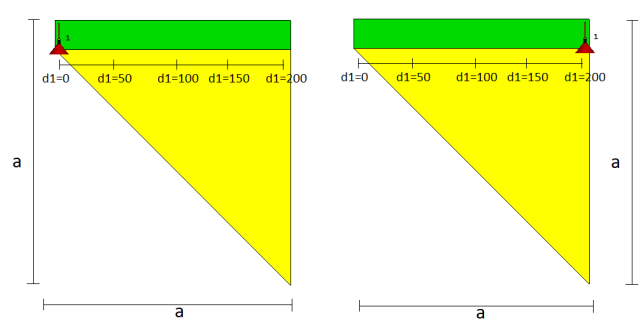

(a)

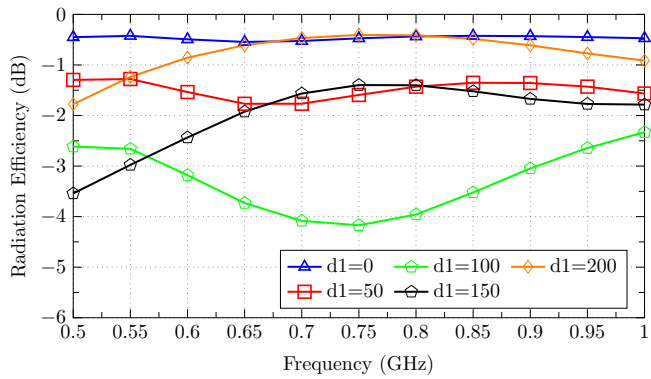

(b)

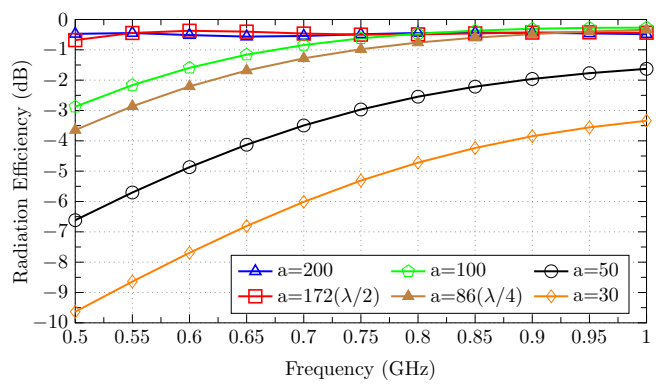

(c)

Fig. 5. a) Left: Triangular PCB with the antenna placed in the corner (d1=0), Right: triangular PCB with the antenna placed in the center $(\mathrm{d} 1=100)$, b)Radiation Efficiency obtained displacing the antenna from $\mathrm{d} 1=0$ until $\mathrm{d} 1=100$ and, c) Radiation efficiency of the antenna in the triangular PCB modifying the side length of the ground plane (a)

The last analysis is based on modifying the length of the PCB (a) and in Fig. 5 (c), the variation of radiation efficiency is depicted. Results confirms the information obtained in the previous designs with a high radiation 
efficiency, which saturates when length a is higher than $\lambda / 2$.

\section{Compact Design with Matching CiRcuit}

A design with a limited ground plane has been analyzed in order to check the viability of the chip antenna solution, and the total efficiency has been calculated. The size of the PCB is $50 \times 50 \mathrm{~mm}^{2}$, with a $=50 \mathrm{~mm}$ smaller than $\lambda / 4$ at 868 $\mathrm{MHz}$. As seen in the previous analysis, when a is smaller than $\lambda / 4$ the efficiency decreases and also the resonance frequency increases. For this design, the terminal line has been modified in order to tune the design at $868 \mathrm{MHz}$.

Due to the size of the ground plane, the real part of the input impedance of the antenna is really low, hence the design is not well match. For this cases, a matching circuit (MC) must be added, and in this case a T-matching circuit has been calculated with real components (Inductors: Murata LQW15AN80 0402, Capacitors: Murata GJM15 0402). In Fig. 6 (a), the analyzed design and the values of the MC are depicted. In Fig. 6 (b) the comparison of the efficiency between the design with and without $\mathrm{MC}$ is shown. Proper total efficiency at the operating frequency $868 \mathrm{MHz}$ when MC is used.

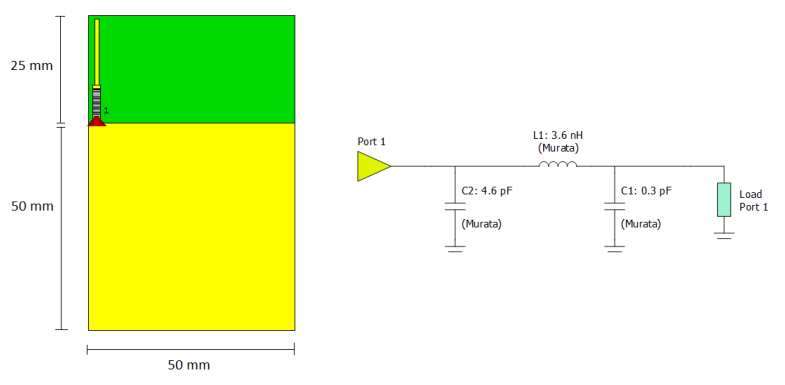

(a)

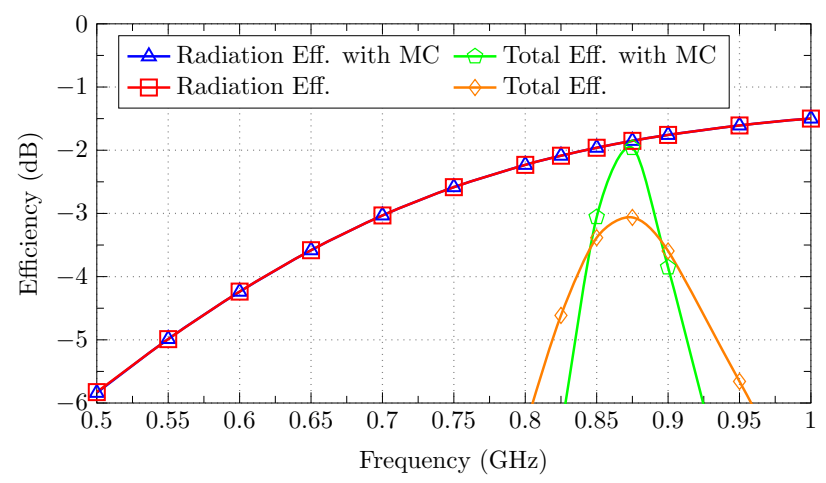

(b)

Fig. 6. a) Right: Square $50 \times 50 \mathrm{~mm}^{2} \mathrm{PCB}$ with the chip antenna in right corner Right: T-Matching circuit, b) Radiation Efficiency and Total Efficiency of the antenna with and without matching circuit(MC)

\section{CONCLUSION}

The study of how the radiation efficiency of a small antenna depending on its position and the shape and size of the ground plane has been done, obtaining key points for managing PCB based solutions with small antennas. It has been demonstrated that the ground plane play a really important role in the radiation and, the design must take into account both parts, the antenna and the ground plane, as an asymmetric monopole.

The first extracted conclusion regarding the position of the antenna is placing the antenna in the corner of any shape is better in terms of radiation efficiency than placing it in the middle of any side.

Two lengths have to be taken into account which interferes directly with the radiation efficiency, the $\lambda / 4$ and $\lambda / 2$ length. It is more important the maximum length left for the current than the shape of the PCB. When any PCB have any side higher than $\lambda / 2$, the radiation efficiency has a high value but does not increase if the ground plane size is enlarged. On the contrary, if the longest side of the $\mathrm{PCB}$ is shorter than $\lambda / 4$ the radiation efficiency is poor and the resonance frequency is greatly affected, shifted toward higher frequencies. In case of a PCB with a length in between $\lambda / 4$ and $\lambda / 2$, the radiation efficiency increases with the size of the PCB until $\lambda / 2$.

\section{ACKNOWLEDGMENT}

This work has been supported by the Spanish Ministry of Science, Innovation, and Universities (Ministerio de Ciencia, Innovación y Universidades) under the FPI research fellowship program (TEC2016-78028-C3-3-P).

\section{REFERENCES}

[1] M. Agiwal, A. Roy, and N. Saxena, "Next generation 5G wireless networks: A comprehensive survey," IEEE Communications Surveys \& Tutorials, vol. 18, no. 3, pp. 1617-1655, 2016.

[2] F. Ferrero, L. Lizzi, C. Danchesi, and S. Boudaud, "Environmental sensitivity of miniature antennas for iot devices," in 2016 IEEE International Symposium on Antennas and Propagation (APSURSI). IEEE, 2016, pp. 1749-1750.

[3] A. R. Chandran, G. A. Conway, and W. G. Scanlon, "Compact slotloaded patch antenna for $868 \mathrm{mhz}$ wireless body area networks," in 2008 Loughborough Antennas and Propagation Conference. IEEE, 2008, pp. 433-436.

[4] K. Diallo, A. NGom, A. Diallo, J.-M. Ribero, I. Dioum, and S. Ouya, "Efficient dual-band pifa antenna for the internet of things (iot)," in 2018 IEEE Conference on Antenna Measurements \& Applications (CAMA). IEEE, 2018, pp. 1-4.

[5] Johanson Technology "https://www.johansontechnology.com".

[6] Antenova "https://www.antenova.com/".

[7] Fractus Antennas "https://www.fractusantennas.com/".

[8] L. Lizzi and F. Ferrero, "Impact of ground plane reduction in antennas for compact terminals," in 2018 2nd URSI Atlantic Radio Science Meeting (AT-RASC). IEEE, 2018, pp. 1-3.

[9] D. Chatterjee and A. K. Kundu, "A small broadband micro strip patch antenna with ground plane optimization," in 2018 IEEE Indian Conference on Antennas and Propogation (InCAP). IEEE, 2018, pp. 1-3.

[10] M. A. ul Haq, S. Koziel, and M. A. Khan, "Systematic study of feed line and ground plane modifications for design of miniaturized wideband antennas," in 2018 IEEE MTT-S International Conference on Numerical Electromagnetic and Multiphysics Modeling and Optimization (NEMO). IEEE, 2018, pp. 1-4.

[11] I. T. Nassar and T. M. Weller, "The ground plane effect of a small meandered line antenna," in WAMICON 2011 Conference Proceedings. IEEE, 2011, pp. 1-5. 